\title{
计算机电子信息技术在工程管理中的应用分析
}

\author{
苏争争 \\ 江苏长天智远交通科技有限公司，江苏 南京 210000
}

[摘要]文章对工程管理中发展电子信息技术的优势进行了分析，提出了实现信息共享、提高信息准确度、强化智能管理三方 面好处。针对目前电子信息技术发展中存在的问题，文章提出了培养专业人才、建设共享平台、发展技术、结合管理环节的 发展措施, 为工程管理提供了一些发展思路。

[关键词]信息共享; 智能化管理; 安全管理

DOI：10.33142/sca.v3i4.2207 中图分类号：TP3;F407.63 文献标识码：A

\section{Application Analysis of Computer Electronic Information Technology in Engineering Management}

SU Zhengzheng

ITSKY, Nanjing, Jiangsu, 210000, China

\begin{abstract}
This paper analyzes the advantages of developing electronic information technology in engineering management, and puts forward the advantages of realizing information sharing, improving information accuracy and strengthening intelligent management. In view of the existing problems in the development of electronic information technology, this paper puts forward the development measures of training professional talents, building a sharing platform, developing technology and combining management links, which provides some development ideas for engineering management.
\end{abstract}

Keywords: information sharing; intelligent management; security management

引言

计算机电子信息技术指的是依托计算机等设备对信息进行收集和处理, 进而形成完备的数据库的技术。电子信息 技术在日常生活中已经十分普及, 给人们的生活带来了很多便利。在工程管理中使用电子信息技术, 也可以有效地提 升工作效率，为工程管理带来了新的发展机遇。

\section{1 在工程管理中应用计算机电子信息技术的意义}

1. 1 实现信息共享

工程管理一般涉及到很多环节和部门, 如安全部门、材料部门、施工设计部门、监管部门等, 多个部门的信息十 分庞杂且彼此之间无法及时沟通交流，造成工程管理流程的不顺畅。利用电子信息技术，可以有效地进行资源共享， 各个部门都可以及时便捷地在数据库中查询到所需要的信息数据, 在一个部门完成工作后, 可以将数据信息上传至云 端, 促进数据库的建立与发展, 大大提升工程管理的效率和水平。

\section{2 提高信息准确度}

利用人工方式进行信息管理主要的问题就在于容易出现人工失误, 对于工程管理这种重视数据完整性与真实性的 行业来说, 人工失误会对后续工作造成严重影响。利用电子信息技术可以有效规避人工工作的失误, 设定好相应的程 序, 系统和软件可以对信息自动进行处理, 处理的速度快, 准确度高, 还可以为数据的完整性提供可靠保证, 满足了 工程管理的需要。

\section{3 加强智能化管理}

技术的进步给各行各业的发展都提出了新的要求, 对数据信息进行智能化管理, 有利于提高数据存储和使用的安 全性。自动化管理是工程管理所追求的目标, 在工作中尽可能避免人工干预, 在降低失误发生的同时还可以极大程度 地解放劳动力, 提升人力资源的利用率, 将重复机械的工作交给计算机去做, 提高基础工作的精准度。智能化管理系 统的建立有利于企业可以更加从容得面对当今市场的激烈竞争, 提升自身的竞争力, 促进自己的技术发展, 为转型技 术型公司奠定基础 ${ }^{[1]}$ 。

\section{2 在工程管理中应用计算机电子信息技术的有效策略}

2.1 培养专业人才, 提高人员水平

计算机电子信息技术的应用需要很高的专业性, 对相关工作人员的素养提出更为严苛的要求。工作人员需要具备 专业的电子信息技术以及掌握相应的操作技能和手段, 但是在实际工作中, 很多工作人员忽略了理论知识的学习, 认 
为只要记住操作步骤就行了。缺乏理论知识的支撑, 工作人员对于技能只是机械记忆步骤, 在面对突发情况时经常手 足无措, 无法采用合适的方法解决问题。针对这种情况, 工程企业需要大力引进专业人才, 让合适的人才去合适的岗 位。对于现有的工作人员, 对于学习意愿较高、接受水平较好的人员, 公司可以安排培训和学习机会, 培养计算机技 术储备军。对于接受水平较差的人员可以安排转岗, 进行管理工作。

在工程管理中，一线的技术的人员过于稀缺，很多工作人员没有将主要精力放在技术的开发和使用上，反而放在 人员管理上, 造成本末倒置的情况。计算机电子信息技术的发展是以技术为驱动的, 所以人才是工作中的核心和灵魂, 重视一线技术人才的培养和引进，对工作人员定期实施考核，确保其技术水平达到工作要求。

\section{2 建设共享平台, 提高安全水平}

信息共享是高效率工作的基础, 建设信息共享平台可以大幅度提高各部门协作的效率, 带来便利性。但需要注意 的是共享平台的建立也十分容易导致信息泄露, 甚至一些政府网站也出现了信息泄露的问题, 如某市政府信息公开网 在发布《优生健康检查人员名单》时, 没有对名单人员的姓名、身份证号和检查日期进行模糊处理, 造成信息泄露, 产生不良影响。还有一些国外的在线项目管理平台将用户的信息完全公开, 造成数据泄露。这些事件对工程管理的电 子共享平台的建立都做出了警醒, 要将安全问题放在平台建立的首要位置, 可以通过建立防火墙、预警系统、及时进 行系统升级等方式加强安全管理, 避免机密信息泄露造成严重后果。

\section{3 推动技术革新}

根据摩尔定律的说法, 计算机更新的周期一般为 18 个月, 电子信息技术的更新是日新月异的，如果企业不能及时 提升自己的技术水平, 将会被同行远远落在后面。工程管理工作中需要各种各样的软件和功能, 基础的办公功能如制 作 PPT、绘制 Excel 表格; 进行工程制图，如 AutoCAD 3DMAX、lightscape 等; 进行工程概预算和财务管理，如 ERP P3 和 P6。为了推动企业电子信息技术的发展就不能满足于当前的技术成果，还要投入大量的人力资金进行创新，不断了 解前沿技术并将其利用在自己的实际工作中，推动工程管理向智能化、信息化的方向不断发展。

\section{4 与工程管理的环节有机结合}

工程管理一般包含多个环节和流程, 例如进度计划管理、质量管理、成本管理、设计管理、技术管理、安全文明 施工管理、环境管理、安全管理等, 要想发挥好计算机电子技术在工程管理中的作用, 就要将电子信息技术与工程管 理的各个环节有机结合起来。

首先, 与工程质量管理的有机结合。做好质量管理有利于提升整个工程的安全性和使用寿命，传统的工程主要依 靠人力的现场勘查后再进行质量汇报, 很容易造成管理中的疏漏或者对于某些情况的考虑不够全面。借用电子信息技 术可以有效地解决这个问题。工作人员在进行质量管理时, 除了现场勘查, 还可以通过绘图软件将建筑情况绘制在系 统中, 全方位地对建筑质量进行考核和计算。还可以搭建一个质量监管平台, 施工人员可以将现场的情况通过照片和 数据表格的形式上传到监督平台上, 方便监督部门了解施工情况, 使监督部门可以及时发现工程中的问题并进行反馈。 各工程团队和企业还可以将工程中出现过的问题上传到数据库里，在其他企业出现类似的问题时可以有据可查。

其次，与工程材料管理有机结合。工程材料的采购和保管是工程进行的基础工作，材料不达标、数量不足等情况 的发生都会使工程延误，造成资源浪费。通过电子信息技术，可以预估所需材料量，形成采购清单，使采购人员可以 按图索瀷, 避免采购的数量不足或超额。在材料入库时可以使用相应的管理软件, 建立入库出库信息表, 在使用前可 以通过信息表的数据了解到材料是否在有效期内，剩余材料如何进行保存等问题，大大提升材料的利用率。

最后, 与工程安全管理有机结合。安全问题是施工现场的关键问题, 许多施工人员为了省事不按照规定的步骤进 行施工, 埋下了安全隐患。通过设置智能监控设备, 可以有效解决这类问题。目前已经有很多厂商推出了适合各类施 工场景的监控设备, 比如红外线监控、烟雾监控、有害气体监控等, 可以在工作人员出现违规操作时发出警告, 通知 后台管理人员进行监督管理。在发生一些意外事故时, 比如有害气体泄露, 可以及时发出警报, 通知现场员工撤离, 自动切断电源, 避免发生二次意外 ${ }^{[2]}$ 。

\section{3 结束语}

“互联网+”的概念在近年来十分火热，对于企业来说，利用好电子信息技术可以有效促进自身发展，提升自身竞 争力。对此, 工程企业应该加大技术的发展力度, 培养专业的信息人才, 不断革新技术。随着 $5 \mathrm{G}$ 时代到来, 更应该紧 随时代步伐，做好信息技术和工程管理的完美融合。

\section{[参考文献]}

[1]马书群.计算机电子信息技术工程管理与应用分析 [J].数字技术与应用, 2019(11): 56-57.

[2] 曾锦源.计算机电子信息技术在工程管理中的应用 [J].数字通信世界, 2020(02) : 186 .

作者简介: 苏争争 (1986.11-), 男, 毕业院校: 江苏食品职业技术学院, 现就职单位: 江苏长天智远交通科技有限公司。 